\title{
Comparison of two surface temperature measurement using thermocouples and infrared camera
}

\author{
Dariusz Michalski ${ }^{*}$, Kinga Strąk, Magdalena Piasecka \\ Faculty of Mechatronics and Mechanical Engineering \\ Kielce University of Technology, Al. 1000-lecia P.P. 7, 25-314 Kielce, Poland
}

\begin{abstract}
This paper compares two methods applied to measure surface temperatures at an experimental setup designed to analyse flow boiling heat transfer. The temperature measurements were performed in two parallel rectangular minichannels, both $1.7 \mathrm{~mm}$ deep, $16 \mathrm{~mm}$ wide and $180 \mathrm{~mm}$ long. The heating element for the fluid flowing in each minichannel was a thin foil made of Haynes-230. The two measurement methods employed to determine the surface temperature of the foil were: the contact method, which involved mounting thermocouples at several points in one minichannel, and the contactless method to study the other minichannel, where the results were provided with an infrared camera. Calculations were necessary to compare the temperature results. Two sets of measurement data obtained for different values of the heat flux were analysed using the basic statistical methods, the method error and the method accuracy. The experimental error and the method accuracy were taken into account. The comparative analysis showed that although the values and distributions of the surface temperatures obtained with the two methods were similar but both methods had certain limitations.
\end{abstract}

\section{Introduction}

Instruments used to measure temperature can be divided into contact and contactless, according to the way the heat is transferred from the body or medium to be measured to the thermometer.

This paper compares the results of temperature measurement realized by contact method based on thermocouples and obtained from infrared thermography, which is an example of a contactless method. In their previous research, the authors performed a comparative analysis for two temperature contactless methods: infrared thermography and liquid crystal thermography [1]. Liquid crystal thermography was used in a study of the FC-72 flow boiling heat transfer in rectangular minichannels with enhanced heated surfaces $[2,3]$.

The thermocouple-based contact method is commonly used for measuring the surface temperature of liquids and gases. In 1821, T. J. Seebeck discovered that it was possible to create an electrical voltage by soldering two different metals (the Seebeck effect). When two metallic elements having different Seebeck coefficients are joined together at one end, a voltage called electromotive force (EMF) can be detected using a voltmeter at the other end [4]. Calibration of thermocouples is very important. The temperature calibration uncertainty can be evaluated, for instance, using the conventional method described in the Guide to the expression of Uncertainty in Measurement (GUM) or the numerical Monte Carlo method [5]. The numerical method is generally more flexible than the conventional method. Characteristic temperatures of type E thermocouples and a simple approximation of the relationship between thermal electromotive force (EMF) and temperature $T$ were discussed in [6]. The characteristic temperatures of the thermocouples were approximated using quadratic polynomials assuming an accuracy of $\pm 0.5 \mathrm{~K}$ over the temperature range from 0 to $1000{ }^{\circ} \mathrm{C}$. Genix et al. [4] describe results obtained with an intrinsic thermocouple used to perform ultra-local temperature measurements. The intrinsic thermocouple, which includes a conducting tip placed in contact with a conducting sample, is designed to measure rapid changes in the surface temperature. A fast-response thermocouple is used to measure, for example, the surface temperature of internal combustion engines, as described by Marr et al. [7]. Heichal et al. [8] discuss the use of thin film sensors to detect a $15 \mathrm{~ns}$ laser pulse.

In the paper by Kus et al. [9] the tool-chip interface temperature was estimated by simultaneously employing a type $\mathrm{K}$ thermocouple and an infrared radiation pyrometer. The finite element method was used to compare the temperature results; the experimental results showed good agreement.

Infrared thermography is a powerful technique that can be used to quantify the thermal field on a measured surface. The characteristic features of the IR camera include quick response time and high sensitivity. The fully two-dimensional IR camera offers colour images and is able to identify surface temperature fields. Errors are easy to detect and correct because of tangential

Corresponding author: michalski@tu.kielce.pl 
distortions. The IR camera is well-suited to study fluid flows that are complex in nature. It can be applied to analyse convective heat fluxes on the heating element or changes in the flow field [10]. Infrared thermography has many applications. Mikielewicz et al. [11] discuss its use to analyse the increase in the dryout heat flux for four fluids flowing through two small-diameter silver tubes. The method has also been found suitable to study the thermo-hydrodynamics of the initial phase of the hydrodynamically fully developed but thermally developing internal laminar flow of water in a square cross-sectional minichannel. Moreover, infrared thermography can be used for high-resolution measurement of the local temperature on the heated wall for one-dimensional conduction in the transverse direction as well as for the determination of the heat transfer coefficient [12].

\section{Experimental stand}

The major parts of the experimental setup presented in figure 1 are: a main loop, a supply and control system and a data and image acquisition system. The flow loop comprised a measurement module (1), an infrared camera (2), a DaqLab 2005 data acquisition station (3), a MCC data acquisition station (SC-1608G) (4) and a PC computer (5). The measurement module with two parallel vertically oriented minichannels (IRT minichannel and TC minichannel), each $1.7 \mathrm{~mm}$ deep, $16 \mathrm{~mm}$ wide and $180 \mathrm{~mm}$ long, was the most important element of the experimental setup.

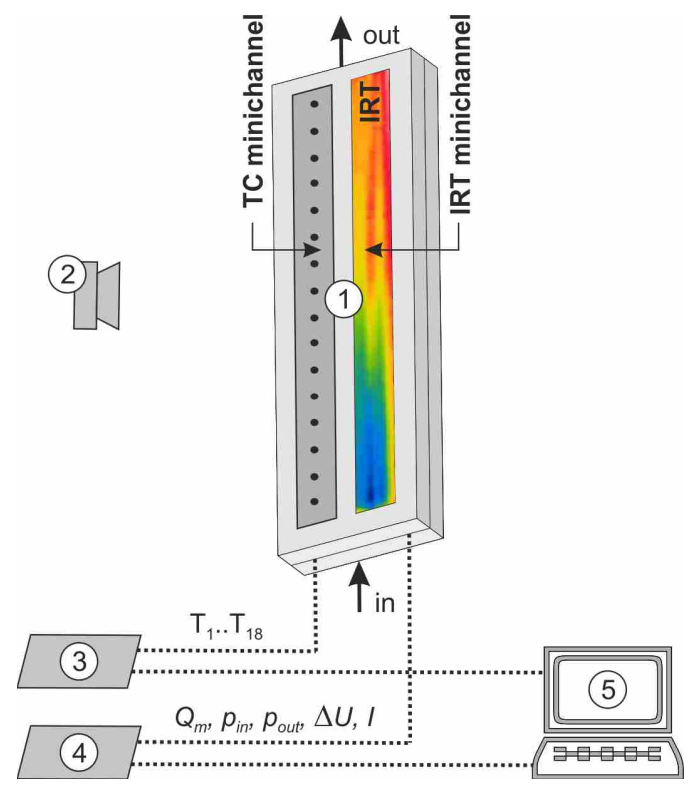

Fig. 1. Schematic diagram of the data acquisition system: 1 - measurement module, 2 - infrared camera, 3 - DaqLab 2005 data acquisition station, 4 - MCC data acquisition station (SC-1608G), 5 - PC computer.

In both minichannels, the heating element was a $0.1 \mathrm{~mm}$ thick foil made of rolled acid-proof Haynes-230 alloy with the surface roughness $R a=0.121 \mu \mathrm{m}$. The working fluid flowing in minichannels was distilled water. In one minichannel (IRT minichannel), the temperature on the outer side of the foil was measured using infrared thermography. The measurements were taken in the central axially symmetric part of the minichannel (4 mm x $180 \mathrm{~mm})$. The surface was coated with a black paint with an emissivity of 0.83 [2]. Narrow micanite panels were placed on both sides of the foil to prevent its deformation. In the other minichannel (TC minichannel), the temperature was monitored by means of thermocouples attached at 16 points on the outer surface of the foil along the central, axially symmetric axis of the minichannel. Two thermocouples were mounted in the minichannel: one at the inlet and the other at the outlet. The signals from the thermocouples were recorded by the data acquisition station.

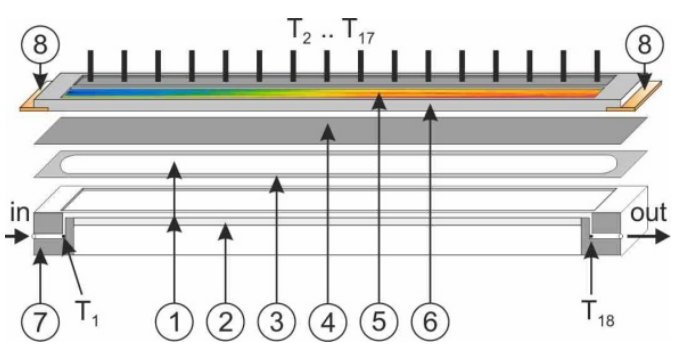

Fig. 2. Schematic diagrams of a measurement module; 1 - minichannel, 2 - glass pane, 3 - PTFE spacer, 4 - heated foil, 5 - IRT thermogram, 6 - front cover, 7 - channel body, 8 - copper electrode.

\section{Two methods of surface temperature measurement}

\subsection{Contact method using thermocouples}

Thermocouples are temperature sensors that respond to a change in the temperature with a change in the thermoelectric force. The thermocouple consists of two conductors differing in material joined together at one end. A thermoelectric effect is used to measure temperature. It consists in creating a thermoelectric force, which is a result of the difference in temperatures between two junctions: the measurement junction (the connected ends of the conductors) and the reference junction (the free ends). The reference temperature is usually fixed at $0{ }^{\circ} \mathrm{C}$. There are different types of thermocouples, each with unique characteristics. The most common are T, E, J, K, N, S, R and B. The types used for high temperatures are G, D and C. Each type has a specific temperature measurement capability so that the results can be represented linearly in the desired measurement range [13].

The thermocouples used in the experiments were manufactured by Czaki Thermo-Product, Poland (type TP-221) with $T_{\max }=+600{ }^{\circ} \mathrm{C}$. They have a NiCr-NiAl type $\mathrm{K}$ sheathed thermocouple sensor with an outer diameter of $0.5 \mathrm{~mm}$. The measurement junction is galvanically isolated from the sheath (type B).

The NiCr-NiAl type $\mathrm{K}$ thermocouple is oxidationproof resistant to higher temperatures, a reducing atmosphere and sulphur compounds. The type $\mathrm{K}$ thermocouple can operate at very high 
temperatures (up to $1000^{\circ} \mathrm{C}$ or even $1100^{\circ} \mathrm{C}$ ); it is more resistant to high temperatures than other thermocouples made of non-noble metals [13].

\subsection{Contactless method by Infrared Thermography}

Infrared thermography is a method that involves the detection of infrared radiation emitted by an object with a temperature higher than absolute zero. Thermal radiation occurs in the region between visible light and microwaves [14].

One of the important parameters in the IR measurement is the emissivity of the object measured $(0 \div 1)$. Emissivity can be defined as the ratio of the radiation emitted by a physical body to the radiation emitted by a black body at the same temperature $[15,16]$.

Infrared cameras produce thermograms, which provide qualitative and quantitative data about the thermal conditions and thermodynamic processes taking place in the observation area. An IR camera can be used to record static conditions or monitor dynamic processes taking place under laboratory conditions. Correct interpretation of thermograms requires knowledge of the properties of the material analysed and the effect of the surroundings. A good knowledge of the measurement technique is also useful as in practice it is often necessary to identify and eliminate the sources of interference $[16,17]$.

\section{Results}

The main aim of this study was to compare the results of the temperature measurements performed simultaneously in two parallel minichannels using two different methods: the contact method (thermocouples - TC) and the contactless method (infrared thermography - IRT).

Piasecka et al. [1] describe a similar analysis, but it was made for two contactless surface temperature measurement methods: infrared thermography and liquid crystal thermography. It was found that the values and distributions of the temperatures obtained with both methods were similar. However, the IR camera used in that study was different from the one employed in this research.

The experiments described in this paper were conducted under constant laboratory conditions with an ambient temperature of $300 \mathrm{~K}$, a relative humidity of $40 \%$ and an atmospheric pressure of $0.095 \mathrm{MPa}$. In the IR measurements, the distance between the IR camera and the foil surface was $0.4 \mathrm{~m}$ and the foil was coated with a black paint with an emissivity of 0.83 .

\subsection{Uncertainty and limitations of thermocouples and IRT}

The type $\mathrm{K}$ thermocouples applied to measure the temperature of the foil surface in one minichannel were $0.5 \mathrm{~mm}$ in diameter. They were capable of measuring temperatures in the range from $-40{ }^{\circ} \mathrm{C}$ to $600{ }^{\circ} \mathrm{C}$. In this study, the measurement accuracy assumed for the type $\mathrm{K}$ thermocouples was about $1.5{ }^{\circ} \mathrm{C}$ in the range from $-40{ }^{\circ} \mathrm{C}$ to $375^{\circ} \mathrm{C}[13,18]$.

The thermocouple-based method has some limitations. One of the major problems is attaching a thermocouple to the surface to be measured. Incorrect bonding may cause the device to show a temperature lower than the actual one. When a thermocouple is used to measure the temperature of an element in a high frequency radio circuit, it may change the electrical characteristics of that circuit.

A thermocouple fixed to a small electronic element may be responsible for the removal of heat from the element. As a result, the temperature displayed is lower than the actual temperature, i.e. one measured before the thermocouple was attached. Decalibration may lead to an error in measurement; it may change the electrical characteristics, which will make the reading inaccurate. Finally, an open thermocouple junction may also cause errors in temperature measurement [19].

The images taken with a FLIR E60 infrared camera system operating in the spectral range from $7.5 \mu \mathrm{m}$ to $13 \mu \mathrm{m}$ were recorded in a digital system with an image frequency of $60 \mathrm{~Hz}$ [20]. As specified by the producer, the FLIR E60 camera has a thermal accuracy of $\pm 2{ }^{\circ} \mathrm{C}$ or $\pm 2 \%$. In an additional certificate, however, we read that a higher accuracy of $\pm 1{ }^{\circ} \mathrm{C}$ or $\pm 1 \%$ can be achieved in the temperature range from 0 to $120^{\circ} \mathrm{C}$ [21].

In the IRT measurements, the effect of the external factors on the measurement accuracy was substantial. It was found that the measurement uncertainty was dependent on several parameters. The most important were the emissivity and moisture of the object, its distance from the camera, the angle of observation, the air supply and, finally, the effect of the sources of interference such as the heat produced by the heating elements or by the lamps lighting the surface.

\section{Comparative statistical analyses of temperature data}

The temperature data obtained for the contact (TC) method and the contactless (IRT) method were compared statistically. Two settings of the increasing heat flux supplied to the foil and three distances from the minichannel inlet were considered. The analysis was performed according to the following procedure:

- graphical comparison of the measurement results concerning the foil temperature (figures 3 and 4);

- determination of the experimental error for the temperature measurement method according to the principles of statistical inference (taking account of the foil temperature) followed by the estimation of the mean value of the experimental error, the estimation of the mean deviations of the experimental error $[22,23]$ and the estimation of the confidence interval for a single experimental error and the measurement accuracy [24]. 


\subsection{Graphical Comparison}

The values of the heated foil temperature measured along the central line in the axially symmetric part of the TC and IRT minichannels were compared graphically. The data obtained by means of thermocouples and infrared thermography for two settings of the increasing heat flux supplied to the foil (denoted as \#1 and \#2) and three distances from the minichannel inlet are shown in figure 3. Figure 4 illustrates differences in the surface temperature of the heated foil $\Delta T$ between the TC and IRT measurements against the time for the selected distances from the minichannels inlet.

\subsection{Basic statistical parameters}

Table 1 provides all the basic statistical parameters used in the comparative statistical analysis of the temperature results obtained from the contact $\mathrm{TC}$ and the contactless IRT methods.

Table 1. Basic statistical parameters

\begin{tabular}{|c|c|c|c|}
\hline \multirow{2}{*}{$\begin{array}{c}\text { Basic parameters } \\
\text { for \#1 }\end{array}$} & \multicolumn{3}{|c|}{ Distances from the minichannels inlet } \\
\cline { 2 - 4 } & $\mathbf{0 . 0 4} \mathbf{~ m}$ & $\mathbf{0 . 0 9} \mathbf{~ m}$ & $\mathbf{0 . 1 4} \mathbf{~ m}$ \\
\hline$M S E$ & 18.45 & 15.01 & 7.97 \\
\hline$M R S E$ & 0.53 & 0.52 & 0.34 \\
\hline$M R A E$ & 0.63 & 0.58 & 0.42 \\
\hline \multirow{2}{*}{$\begin{array}{c}\text { Basic parameters } \\
\text { for \#2 }\end{array}$} & Distances from the minichannels inlet \\
\cline { 2 - 4 } & $\mathbf{0 . 0 4} \mathbf{~ m}$ & $\mathbf{0 . 0 9} \mathbf{~ m}$ & $\mathbf{0 . 1 4} \mathbf{~ m}$ \\
\hline$M S E$ & 11.96 & 19.25 & 8.87 \\
\hline$M R S E$ & 0.42 & 0.59 & 0.37 \\
\hline$M R A E$ & 0.55 & 0.73 & 0.45 \\
\hline
\end{tabular}

where: MSE -Mean Squared Error, MRSE - Mean Relative Squared Error, $M R A E$ - Mean Relative Absolute Error.

Two settings of the heat flux supplied to the foil $(\# 1, \# 2)$ and three distances from the minichannel inlet were considered. The statistical parameters under analysis were: the mean squared error (MSE), the mean relative squared error (MRSE), and the mean relative absolute error $(M R A E)$ [25].

\subsection{Relative experimental error}

The investigations included determining the measurement accuracy of both methods. As IRT was assumed to be the reference method, the experimental results obtained with the thermocouples were compared to those recorded with the infrared camera $[23,24]$.

The relative experimental error (EME) was calculated using the following relationship [22]:

$$
E M E=\frac{T_{T C}-T_{I R T}}{T_{I R T}},
$$

where: $T_{T C}$ - foil temperature obtained with the thermocouples (the test method), $T_{I R T}$ - foil temperature measured by IRT (the reference method).
The IRT method was selected to be the reference method for the measurement of the foil temperature because the measurement error obtained for IRT was lower [16] than that reported for TC $[13,18]$. The relative error of the TC (test) method is shown below.
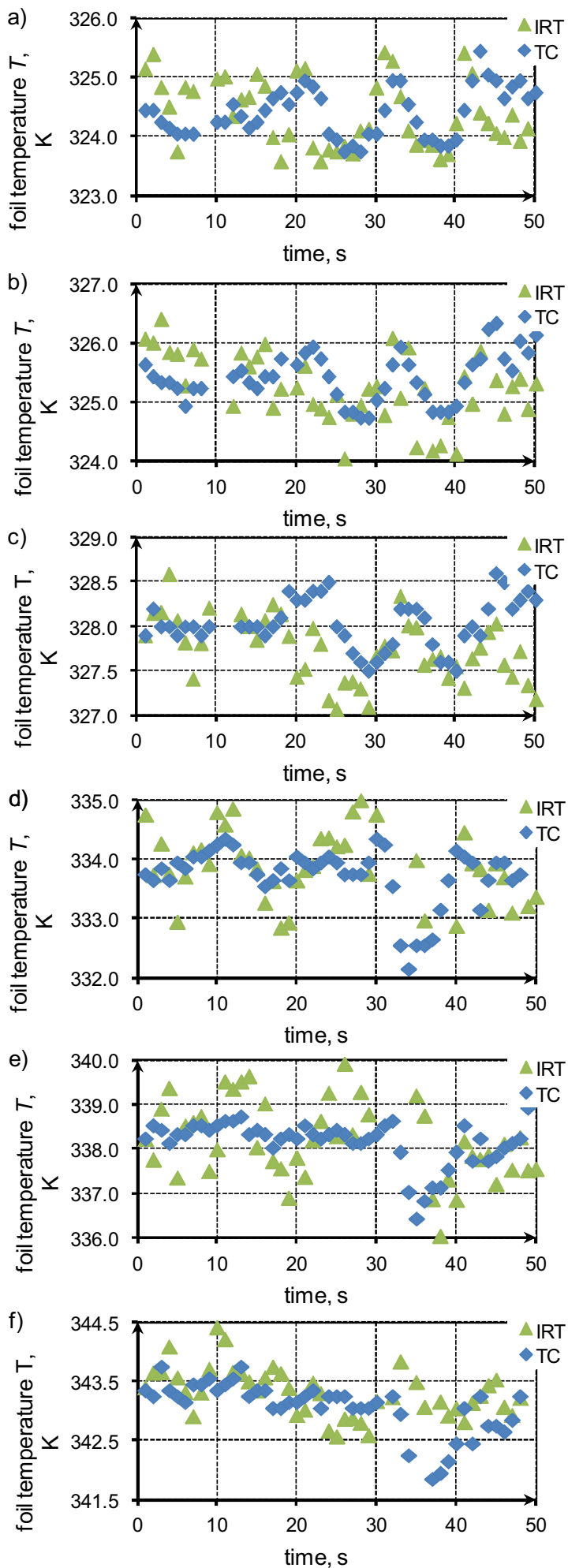

Fig. 3. Heated foil temperature vs. time for the selected distances from the minichannels inlet: a, d) $0.04 \mathrm{~m}, \mathrm{~b}$, e) 0.09 $\mathrm{m}$ and c, f) $0.14 \mathrm{~m}$; data obtained from IRT (green points) and TC (blue points); settings of the heat flux: a-c) \#1, d-f) \#2. 

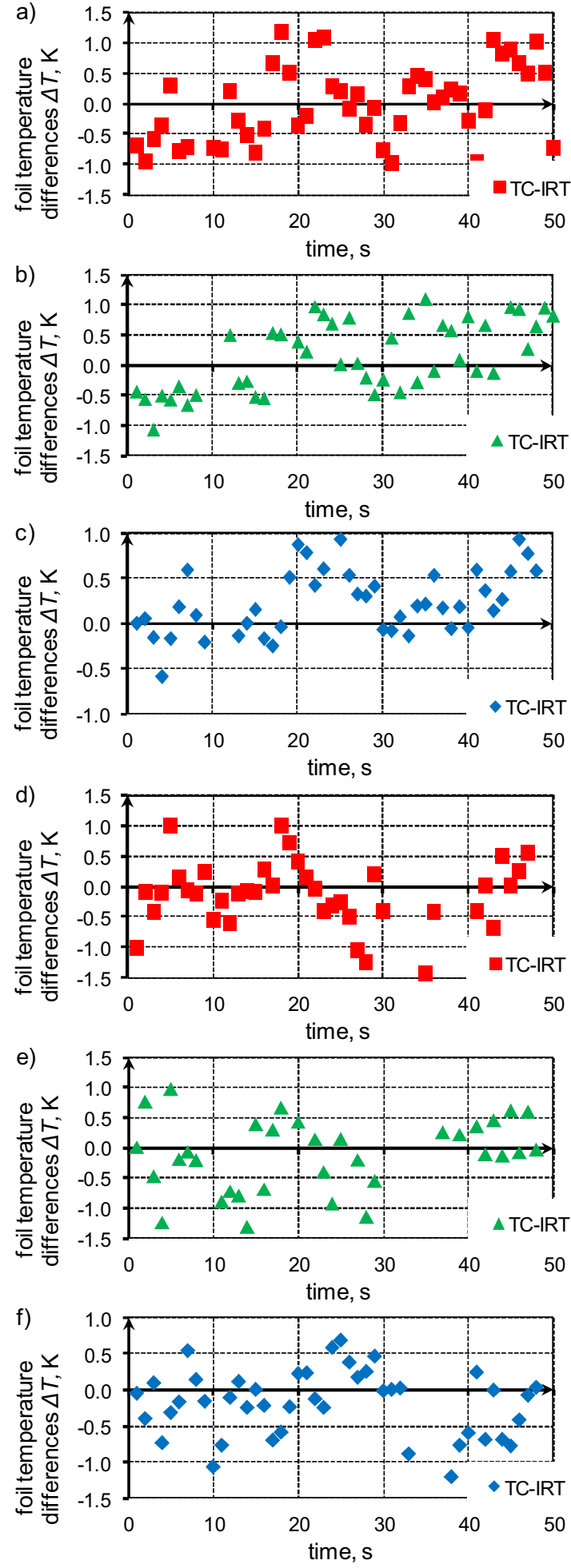

Fig. 4. Differences in the surface temperature of the heated foil between the TC and IRT measurements vs. time for the selected distances from the minichannels inlet: a, d) $0.04 \mathrm{~m}$ (red points), b, e) $0.09 \mathrm{~m}$ (green points) and c, f) $0.14 \mathrm{~m}$ (blue points); data obtained from IR (green points) and TC (blue points); settings of the heat flux: a-c) \#1, d-f) \#2.

The mean value of the experimental error $(\overline{E M E})$ and the confidence interval $(C I)$ for the mean value of the experimental error were provided in [1]. In the analysed population, $\overline{E M E}$ was estimated using the following procedure [24]: determine the experimental errors for the TC and IRT methods, calculate the mean values of the experimental errors and determine the intervals of confidence for the mean values of the experimental errors with normal distribution.

\subsection{Accuracy of the TC and IRT methods}

The values of the experimental errors were used to estimate the accuracy of the methods. The calculations were based on the following relationship:

$$
M A=\left|\overline{E M E} \pm u_{p} \cdot s\right|_{\max } \cdot 100 \%,
$$

where: $\overline{E M E}-$ mean value of the relative experimental error, $u_{p}$ - the expansion coefficient for the level of significance $\alpha=0.05, u_{p}=2$ [26], and $s$-mean deviation of the experimental error.

The method accuracy (MA) determined with Eq. (2) [22] was used to qualitatively assess the measurement accuracy of the Czaki Thermo-Product TP-221 type $\mathrm{K}$ thermocouples employed in the tests.

Table 2 shows results of the statistical analysis including the relative experimental error obtained by comparing the TC and IRT methods.

Table 2.Method accuracy and basic statistical parameters of the experimental error

\begin{tabular}{|c|c|c|c|}
\hline \multirow{2}{*}{$\begin{array}{c}\text { Basic parameters } \\
\text { for \#1 }\end{array}$} & \multicolumn{3}{|c|}{ Distances from the minichannels inlet } \\
\cline { 2 - 4 } Sample size $n$ & $\mathbf{0 . 0 4} \mathbf{~ m}$ & $\mathbf{0 . 0 9} \mathbf{~ m}$ & $\mathbf{0 . 1 4} \mathbf{~ m}$ \\
\hline Min $E M E$ & -0.0030 & -0.0033 & -0.0018 \\
\hline Max EME & 0.0036 & 0.0034 & 0.0041 \\
\hline Median & -0.00011 & 0.00028 & 0.00058 \\
\hline \hline EME & -0.000020 & 0.00047 & 0.00089 \\
\hline$s$ & 0.0019 & 0.0018 & 0.0013 \\
\hline CI & 0.00002 & 0.00047 & 0.00089 \\
& \pm 0.0038 & \pm 0.0036 & \pm 0.0026 \\
\hline$M A$ & $0.39 \%$ & $0.41 \%$ & $0.34 \%$ \\
\hline Basic parameters & Distances from the minichannels inlet \\
\cline { 2 - 4 } for \#2 & $\mathbf{0 . 0 4} \mathbf{~ m}$ & $\mathbf{0 . 0 9} \mathbf{~ m}$ & $\mathbf{0 . 1 4} \mathbf{~ m}$ \\
\hline Sample size $n$ & 40 & 38 & 43 \\
\hline Min $E M E$ & -0.0043 & -0.0057 & -0.0035 \\
\hline Max $E M E$ & 0.0030 & 0.0029 & 0.0020 \\
\hline \hline$E M E$ & -0.00048 & -0.00071 & -0.00057 \\
\hline Median & -0.00031 & -0.00033 & -0.00042 \\
\hline$S$ & 0.0017 & 0.0022 & 0.0013 \\
\hline CI & -0.00048 & -0.00071 & -0.00057 \\
\pm 0.0034 & \pm 0.0044 & \pm 0.0026 \\
\hline$M A$ & $0.38 \%$ & $0.50 \%$ & $0.33 \%$ \\
\hline
\end{tabular}

where: $E M E$ - experimental error, $\overline{E M E}$ - mean values of the relative experimental error, $s-$ mean deviation, $C I$ - confidence interval, $M A-$ method accuracy.

From the method accuracy (MA) it is evident that the two methods used for the measurement of the foil temperature are similar. In certain ranges, they can be used interchangeably. Under laboratory conditions, the maximum accuracy of the measurement system can reach $15 \%[22,23]$. In the case considered, the 
maximum value of $M A$ was $0.5 \%$, which is very low when compared with the literature data [22, 23].

\subsection{Results and discussion}

Comparing the time-temperature curves in figures 3 a-c obtained for heat flux $\# 1$, we can see that the foil temperature measured with thermocouples is similar to that obtained with IRT. However, the curve created for the TC method is smoother. Since IRT is more sensitive to changes in the foil temperature, the scatter of results was greater. The curves obtained for TC and IRT are similar in shape.

Comparing the time-temperature curves in figures 3 d-f, obtained for heat flux \#2, we can notice that the foil temperature measured with the TC method is similar to that obtained with IRT. As thermocouples are less sensitive to changes in the foil temperature, the curves are smoother. Moreover, there are no local or global extremes as is the case of curves created on the basis of the IRT results. The differences between the TC and IRT curves are more visible for heat flux \#1. The maximum differences in the surface temperature of the heated foil (figure 4) between heat fluxes \#1 and \#2 are less than $3 \mathrm{~K}$ The differences are acceptable for the experimental error obtained for the thermocouples $[13,18]$ and the infrared camera [21].

As can be seen from Table 1, the values of the mean squared error (MSE) are relatively high. However, the values of the mean relative squared error (MRSE) and the mean relative absolute error (MRAE) are rather low. The values of the median provided in this table are lower than the average in all the cases considered. The values of the standard deviation are high, which may indicate difficult experimental conditions - presence of a large number of sources of disturbance affecting the measurement results. The tests were continued after some structural modifications had been made to the experimental setup, which enabled elimination of most sources of disturbance.

\section{Conclusions}

The paper has compared two methods for measuring the surface temperature of the heated foil. The experiments were conducted using a special test stand designed to analyse fluid boiling heat transfer in parallel minichannels. Two methods were employed: contact and contactless. The surface temperature of the heated foil in one minichannel was measured with thermocouples (the contact method). The foil temperature in the other was determined using infrared thermography (the contactless method). Two sets of measurement data obtained for different values of the heat flux were analysed by applying the basic statistical method, the experimental error and the method accuracy. The comparative analysis indicated that the values of the foil temperature obtained with the two methods were similar.

\section{References}

1. M. Piasecka, D. Michalski, K. Strąk, EPJ Web of Conf. 114, paper 02094 (2016)

2. M. Piasecka, K. Strąk, B., Heat Transf. Eng. 38 (2017) (to be published)

3. M. Piasecka, Int. J. Refrig. 56, 198-212. (2015)

4. M. Genix, P. Vairac, B. Cretin, Int. J. Therm. Sci. 48, 1679-1682 (2009)

5. J. V. Pearce, P.M. Harris, J.C. Greenwood, 31, 1517-1526 (2010)

6. V.A. Drebushchak, Thermochim. Acta. 603, 218 226 (2015)

7. M.A. Marr, J.S. Wallace, S. Chandra, L. Pershin, J. Mostaghimi, Exp. Therm. Fluid Sci. 34, 183-189 (2010)

8. Y. Heichal, S. Chandra, E. Bordatchev, Exp. Therm. Fluid Sci. 30, 153-159 (2005)

9. A. Kus, Y. Isik, M. Cemal Cakir, S. Coşkun, K. Özdemir, Sensors (Switzerland) 15, 1274-1291 (2015)

10. G.M. Carlomagno, G. Cardone, Exp. Fluids. 49, 1187-1218 (2010)

11. D. Mikielewicz, J. Wajs, M. Gliński, A.B.R.S. Zrooga, Exp. Therm. Fluid Sci. 44, 556-564 (2013).

12. B. Mehta, S. Khandekar, Exp. Therm. Fluid Sci. 42, 219-229 (2012)

13. https://www.czaki.pl/en/catalogue

14. A. Rogalski, K. Chrzanowski, Optoelectron. Rev. 10, 653-691 (2002)

15. T. Karwat, Insulations 5, 33-36 (2008) (in Polish)

16. K. Ziętala, Application of infrared camera in civil engineering, Proc. 11th Eur. Conf. Young Res. Sci. Work. Transcom 2015, Žilina, 347-352 (2015)

17. M. Pešek, O. Pech, EPJ Web Conf. 67, paper 02091 (2014)

18. PN-EN 60584-1:2014-04 - Termoelementy Czesść 1: Specyfikacje i tolerancje EMF

19. B.R.J. Rogers, I.T.C. Senior, T. Course, Inframation 3, 10-12 (2002)

20. http://www.flir-direct.com

21. Calibration certificate No. K1501035, Calibration laboratory No. 2372, accredited by Czech accreditation Institute under ČSN EN ISO/IEC 17025:2005 for: Calibration of non-contact temperature measuring instruments

22. S. Adamczak, D. Michalski, Metrol. Meas. Syst. 11, 221-234 (2004)

23. S. Adamczak, Measurement of surface texture. Form profiles, waviness and roughness (in Polish), (WNT, Warsaw, Poland, 2008)

24. M.L. Tiku, Aust. J. Stat. 22, 260-275 (1980)

25. G. Bohm, Introduction to Statistics and Data Analysis for Physicists (Verlag Deutsches Elektronen-Synchrotrofn, 2010)

26. ISO 10012:2003 Measurement management systems -Requirements for measurement processes and measuring equipment 